\title{
Influence of stress anisotropy on stress distributions in gap-graded soils
}

\author{
Marion Artigaut ${ }^{1}$, Adnan Sufian ${ }^{1}$, Xiaoxiao Ding ${ }^{1}$, Tom Shire $^{2}$, and Catherine O'Sullivan ${ }^{1 *}$ \\ ${ }^{1}$ Imperial College London, Civil and Environmental Engineering Department, London, United Kingdom \\ ${ }^{2}$ University of Glasgow, School of Engineering, Glasgow, United Kingdom
}

\begin{abstract}
The behaviour of gap graded soils comprising non-plastic fines (sand or silt) mixed with a coarser sand or gravel fraction has received attention from researchers interested in internal instability under seepage loading (a form of internal erosion) as well as researchers interested in load:deformation responses. Skempton and Brogan [1] postulated that resistance to seepage induced instability depends upon the proportion of the overall applied stress that is transmitted by the finer fraction. Shire et al. [2] explored Skempton and Brogan's hypothesis using DEM simulations to look at the proportion of the applied stress transmitted by the finer fractions $(\alpha)$ in ideal isotropic samples. They showed that at low fines contents $\left(\mathrm{FC}<F C^{*}\right)$ the average stress transmitted by the finer grains is less than the applied stress $(\alpha<1)$, while for $\mathrm{FC}>F C^{+}$the fines play a key role in stress transmission $(\alpha>1)$; for $\mathrm{FC}^{*}<\mathrm{FC}<\mathrm{FC}^{+}, \alpha$ depends on the sample density. The current contribution describes a series of constant $p^{\prime}$ DEM triaxial test simulations carried out to assess the evolution of stress heterogeneity with shearing. The simulation data generated indicate that a sample can transition from being fines dominated (with the fines transmitting a significant proportion of the applied stress and $\alpha$ $\geq 1$ ) to coarse or sand- dominated (with $\alpha<1$ ) as the material dilates during shear deformation. While $\alpha$ reduces as the samples dilate, the relationship between the $\alpha$ and the sample void ratio is non-trivial. The anisotropy of the coarse-coarse contact network exceeds the overall contact force anisotropy; this indicates that the deviator stress is transmitted through a strong force network passing through the coarse-coarse contacts supported by the fine-coarse contacts.
\end{abstract}

\section{Background}

As outlined by Zuo and Baudet [3] the influence of the fines content $(F C)$ on the behaviour of gap-graded soils containing non-plastic fines has attracted interest from researchers considering load:deformation response as well as researchers considering seepage-induced internal instability or suffusion (e.g. Skempton and Brogan [1]

Amongst researchers considering the influence of $F C$ on load:deformation response, it is accepted that there is a transitional fines content, $F C_{t}$, that demarks the boundary between materials whose behaviour is dominated by the coarser fraction ("sand dominated") and materials whose behaviour is dominated by the finer fraction ("fines dominated") (Zuo and Baudet [3]). Zuo and Baudet describe various approaches that have been used to determine $F C_{t}$ and showed that determining a unique $F C_{t}$ is non-trivial: different approaches give different $F C_{t}$ values. Skempton and Brogan [1] considered the resistance of gap graded soils to internal instability (suffusion), i.e. the preferential erosion of the finer grains at hydraulic gradients lower than the Terzaghi critical hydraulic gradient $(i \approx 1)$. Rather than seeking a unique $F C_{t}$, Skempton and and Shire et al. [2] recognised that there is a $F C^{*}$ value beneath which the fines do not fill the void space (i.e. an underfilled fabric) between the coarse grains, while there is a second $\mathrm{FC}^{+}$value above which the fines separate the coarse grains (i.e. an overfilled fabric). Skempton and Brogan [1] inferred the proportion of the applied stress carried by the finer grains $(\alpha)$ by considering the hydraulic gradient that induced fines migration in their rigid wall permeameter experiments. For an underfilled material (sand dominated) $\alpha<1$, while $\alpha \geq 1$ implies an overfilled material (fines dominated). For their DEM simulations, Shire et al. [2] directly calculated $\alpha$ by considering the contact forces and showed that for $F C^{*}<F C<F C^{+}$, $\alpha$ depends on the void ratio $(e)$ and relative size of fines and coarse particles, i.e. their data do not support the existence of a unique $F C_{t}$ value.

The current contribution extends the study of Shire et al. by considering how $\alpha$ varies during triaxial shearing. The objectives are (1) to establish whether a fines dominated material can evolve to become sand dominated due to shear induced dilation; and (2) to examine how the relationship between $\alpha$ and $e$ is influenced by stress and fabric anisotropy for $F C \approx F C^{*}$ and $F C \approx F C^{+}$.

\section{Simulation approach}

The simulations followed the approach used by Shire et al.[2], where each sample was created by isotropically compressing a non-contacting cloud of spherical particles

* Corresponding author: cath.osullivan@imperial.ac.uk 
to a mean effective stress $\left(p^{\prime}\right)$ of $50 \mathrm{kPa}$ using periodic domains and neglecting gravity. The current study extended the analyses to consider the evolution of the distribution in $\alpha$ during constant $p^{\prime}$ triaxial shearing. Two particle size distributions (PSDs) were considered, as illustrated in Figure 1, with FCs of $25 \%$ and $35 \%$, corresponding to $\mathrm{FC}^{*}$ and $\mathrm{FC}^{+}$respectively. These $\mathrm{FC}$ values were also considered in the coupled DEM-CFD (Computational Fluid Dynamics) simulations of Kawano et al. [4] Three density values were considered for each FC, which was controlled by varying the coefficient of friction used during isotropic compression. In the current study, each sample was isotropically compressed to 100 $\mathrm{kPa}$ and then subjected to a constant $p^{\prime}$ triaxial compression test using a servo-controlled algorithm as outlined in Thornton [5] and implemented in the LAMMPS code $[6,7]$. Tables 1 and 2 summarize the DEM samples and input parameters, respectively. A simplified Hertz-Mindlin contact model was used. Each simulation was terminated at an axial strain of $4 \%$.

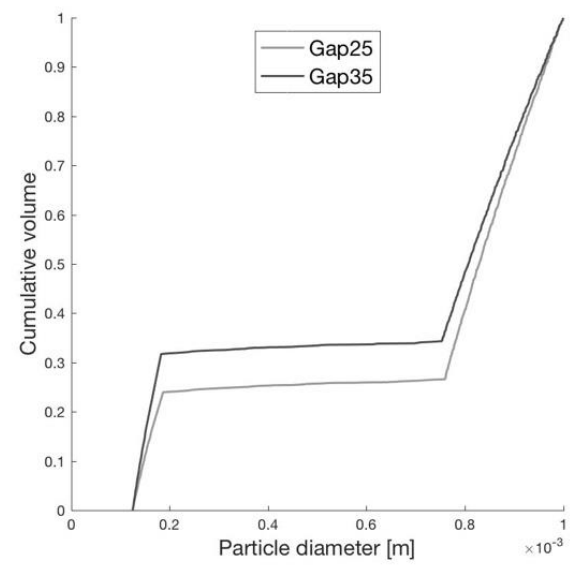

Fig. 1. Particle size distribution of samples

Table 1. Summary of virtual DEM samples $\left(e_{0}=e\right.$ after isotropic compression to $100 \mathrm{kPa} ; \mu_{\text {compression }}=$ coefficient of friction used during isotropic compression)

\begin{tabular}{|l|c|c|c|c|}
\hline \multicolumn{1}{|c|}{ Sample } & $\begin{array}{c}\text { No of } \\
\text { particles }\end{array}$ & $\boldsymbol{F C}$ & $\boldsymbol{e}_{\boldsymbol{0}}$ & $\boldsymbol{\mu}_{\text {compression }}$ \\
\hline Gap 25 loose & 36,950 & $25 \%$ & 0.344 & 0.3 \\
\hline Gap 25 medium & 36,950 & $25 \%$ & 0.283 & 0.1 \\
\hline Gap 25 dense & 36,950 & $25 \%$ & 0.261 & 0.0 \\
\hline Gap 35 loose & 50,357 & $35 \%$ & 0.324 & 0.3 \\
\hline Gap 35 medium & 50,357 & $35 \%$ & 0.317 & 0.1 \\
\hline Gap 35 dense & 50,357 & $35 \%$ & 0.287 & 0.0 \\
\hline
\end{tabular}

Table 2. Summary of DEM simulation parameters

\begin{tabular}{|l|c|}
\hline \multicolumn{1}{|c|}{ Parameter } & Value \\
\hline Particle density & $2670 \mathrm{~kg} / \mathrm{m}^{3}$ \\
\hline Poisson's ratio & 0.2 \\
\hline Shear modulus & $25 \mathrm{GPa}$ \\
\hline $\begin{array}{l}\text { Coefficient of friction during } \\
\text { constant } p \text { ' triaxial } \\
\text { compression }\end{array}$ & 0.3 \\
\hline Local damping coefficient & 0.05 \\
\hline
\end{tabular}

The macroscopic responses of the samples are shown in Figure 2. In all cases there are measurable fluctuations in the load:deformation response (Figure 3(a)); these are indicative of a small sample. Barreto [8] showed that for a mono-disperse material a representative response can be achieved using 5,000 grains. The results show that for gap graded materials significantly larger samples are required to obtain a smooth response. There are significantly more contacts engaged in the Gap35 samples (140,500-288,040 after isotropic compression) in comparison with the Gap25 samples $(55,425-198,420$ after isotropic compression), consequently the Gap35 samples tend to have less fluctuations than the Gap25 samples.

The responses observed are in line with our understanding of soil behaviour; the dense samples are stiffer and more dilatant than the loose samples. A clear peak shear stress is mobilized at relatively small strains in the dense and medium samples, while it is not clear that the loose samples have achieved their peak stress over the strain interval considered. It is clear that neither sample is at a critical state; arguably the volumetric response on Figure 2(b) could be extrapolated to identify critical state $e, p^{\prime}$ values for the Gap 35 samples, however it is less clear that the Gap 25 samples converge to a specific point.

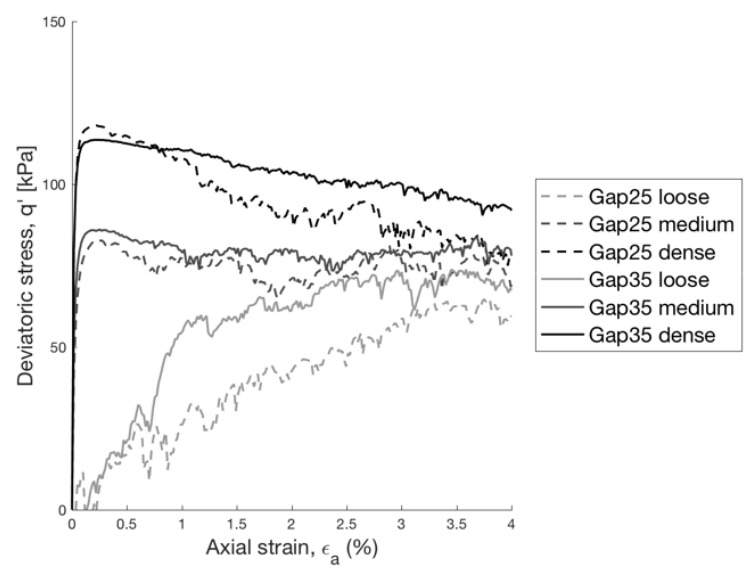

a) Deviator stress $(q)$ versus axial strain

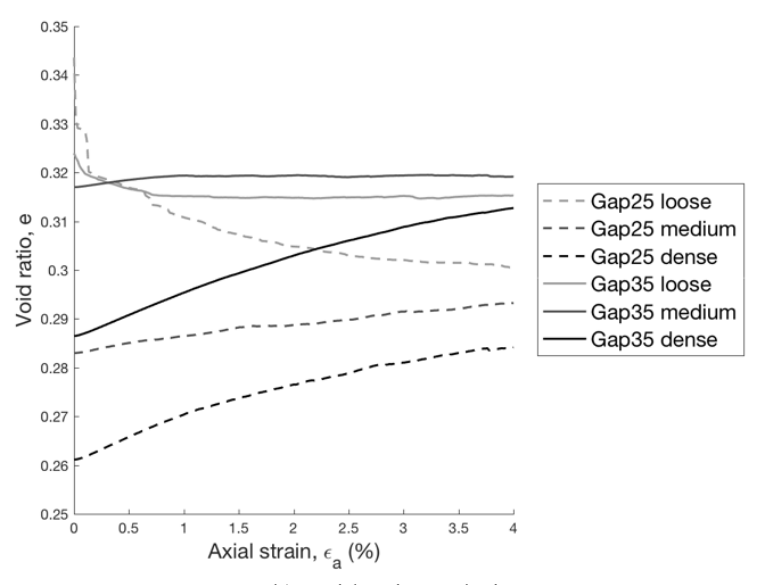

b) Void ratio evolution

Fig. 2. Macroscopic response of the samples

\section{Evolution of stress inhomogeneity}

The stress reduction factor, $\alpha$, is the ratio of the mean stress carried by the finer fraction $p_{f}^{\prime}$ of a sample to the overall stress applied to the sample 


$$
\alpha=p_{f}^{\prime} / p^{\prime}
$$

Following Shire et al. (2014)

$$
p_{f}^{\prime}=\frac{(1-n)}{\sum_{i=1}^{N_{p, f}} V^{i}} \sum_{i=1}^{N_{p, f}}\left(p^{i} V^{i}\right)
$$

where $n$ is the sample porosity, $N_{p, f}$ is the number of fine particles, $V^{i}$ is the volume of particle $i, p^{i}$ is the mean stress in particle $i$ (calculated considering the contact forces and their positions following Potyondy and Cundall [9]).

Shire et al. [2] took $\alpha$ to be a measure of the risk of internal instability; a low stress reduction factor represents a high risk of seepage-induced internal instability. However, taking a more general perspective, $\alpha$ quantifies the extent to which the finer grains are involved in the stress transmission in the sample and contributing to the overall load:deformation response. Where $\quad \alpha<1$ the material is underfilled (sand dominated), while $\alpha \geq 1$ implies an overfilled material (fines dominated).

Referring to Figure 3 it is clear that $\alpha$ varies significantly during shearing, particularly for the dense samples. Most notably there is a reduction in $\alpha$ from 0.72 to 0.22 for sample Gap25 dense where $\mathrm{FC} \approx F C^{*}$. This represents a significant evolution in the role the fine grains play in stress transmission as the sample is sheared. The reduction in $\alpha$ for sample Gap35 dense (with FC $\approx$ $F C^{+}$) is less marked (from 1.15 to 0.69 ) but still significant. The loose samples for Gap25 and Gap35 exhibit minimal changes in $\alpha$ during shearing. The $\alpha$ values for the Gap25 samples converge during shearing to values close to 0.2 ; however there is no indication of convergence for the Gap35 samples.

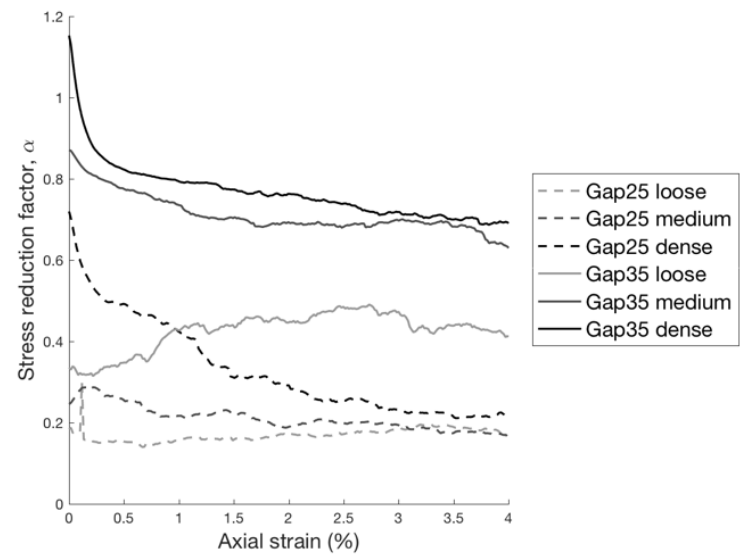

Fig. 3. Evolution of stress reduction factor $(\alpha)$ with shearing

For their isotropic samples Shire et al. [2] showed that for $F C^{*}<F C<F C^{+}, \alpha$ depends on sample density and so $\alpha$ was plotted against $e$ to assess whether the shear-induced variation in $\alpha$ can simply be related to shear-induced volumetric strain. Figure 4 suggests this is the case for the Gap25 samples. The dense and medium sample responses effectively overlap and a smooth curve can be drawn through the data. (The fluctuation at $e=0.33$ values for the loose sample is a sample size artefact.) For the Gap35 samples the $\alpha-e$ relationship is more complex.

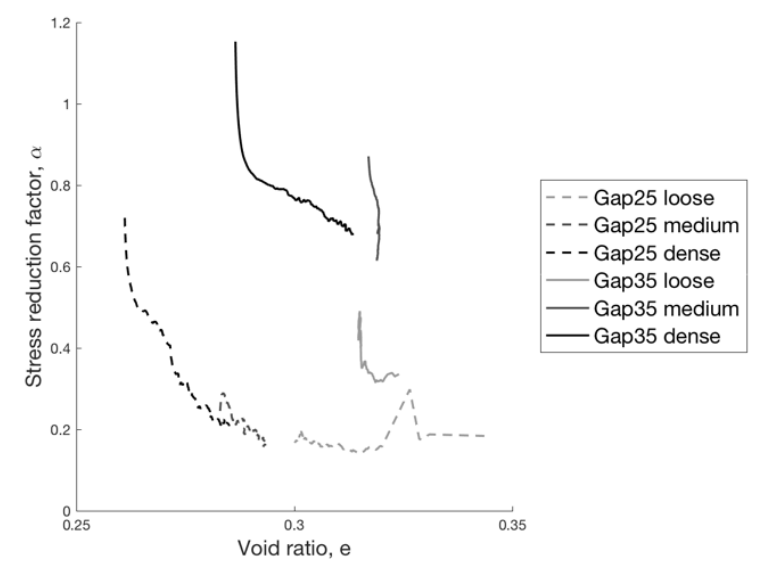

Fig. 4. Variation in stress reduction factor $(\alpha)$ with void ratio $(e)$

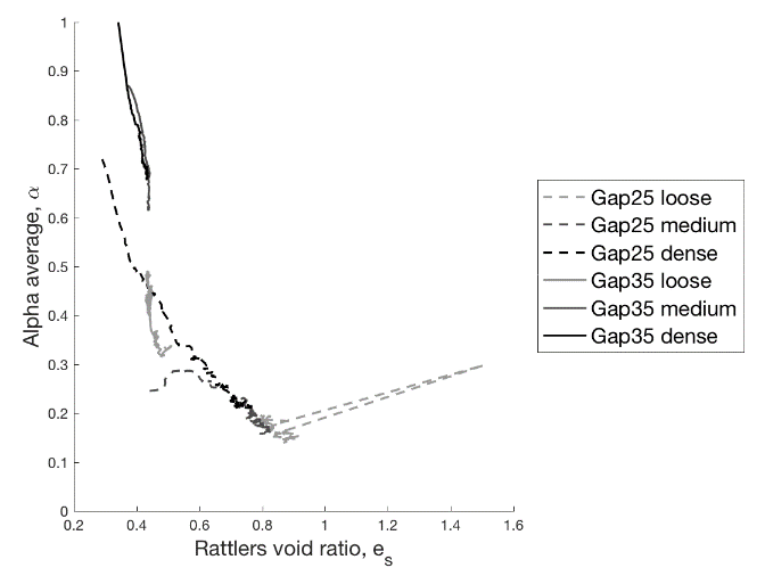

Fig. 5. Variation of stress reduction factor $(\alpha)$ with rattler void ratio $e_{s}$

As outlined in Thevanayagam et al.[10], alternative definitions of void ratio (intergranular and interfine void ratios) can be used to gain insight into the response of gapgraded materials. Various alternative definitions of $e$ were considered here including the rattlers void ratio $\left(e_{s}\right)$, which considers particles with 1 or no carrying contacts as part of the void volume. Figure 5 shows that for both PSDs, there is a consistent $\alpha-e_{s}$ relationship.

The $\alpha$ parameter is an indirect measure of the partitioning of the forces between the coarse and fine grains. A more direct understanding of force transmission can be obtained by classifying each contact into one of three subsets: coarse-coarse contacts, fine-fine contacts and coarse-fine contacts (where coarse-coarse denotes contact between two coarse particles, etc.). Figure 6 illustrates the proportion of the deviator stress $(q)$ transmitted by each of these subsets for all 6 samples considered here. For the dense samples, an increase in the fraction of stress carried by the coarse-coarse contacts can be seen, while the stress carried by the coarse-fine and fine-fine contacts decreases as the sample is sheared. In the loose samples following the initial response, there is a less consistent evolution with the proportion of stress carried by both the 

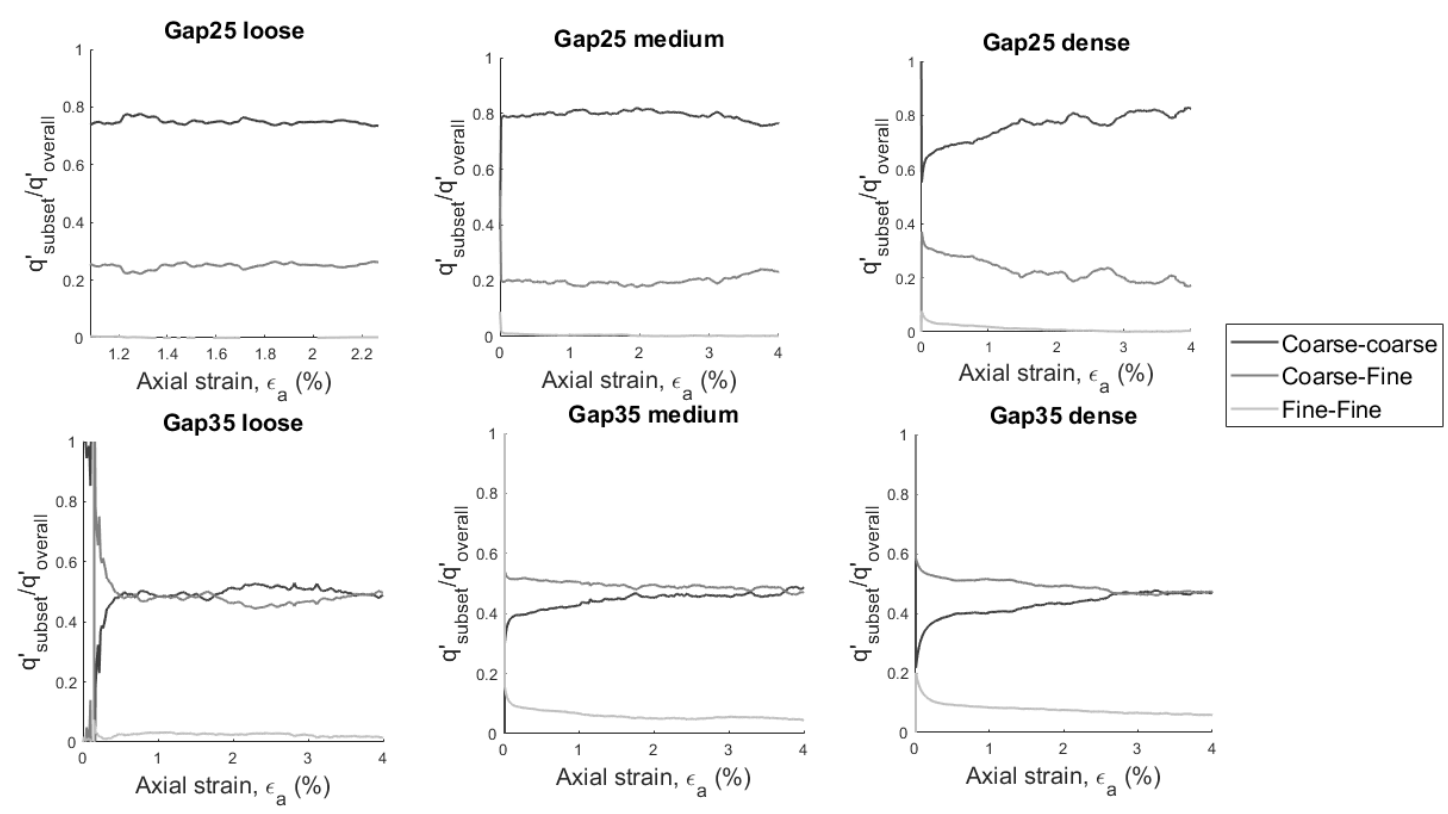

Fig. 6. Distribution of overall deviator stress amongst coarse-coarse, coarse-fine and fine-fine contacts

coarse-coarse and coarse-fine contacts remaining approximately constant during shearing. The fine-fine contacts have a non-negligible contribution to $q$ only in the Gap35 medium and dense samples. This suggest that it is only in these samples that there are sufficient fine particles to create an overfilled fabric (as described in Shire et al. (2014)) or the case (iii) and (iv) fabrics proposed by Thevanayagam et al. [10].

\section{Evolution of fabric anisotropy}

To assess the anisotropy of the samples, a useful tool is the fabric tensor $(\Phi)$.

$$
\phi_{i j}=\frac{1}{N_{c}} \sum_{k=1}^{N_{c}} n_{i}^{k} n_{j}^{k}
$$

where $n_{i}^{k}$ is the contact normal vector for contact $k$. From this fabric tensor, one can calculate the deviatoric fabric. It is a measure of the bias in any preferential alignment of contacts; the deviatoric fabric considered here is given by

$$
\phi_{d e v}=\phi_{z z}-\frac{1}{2}\left(\phi_{x x}+\phi_{y y}\right)
$$

which accounts for axisymmetry in the triaxial sheared samples. Figure 7(a) considers the evolution of $\phi_{d e v}$ during shear deformation, where $\phi_{d e v}$ is calculated by considering all contacts in the sample. The fabric anisotropy increases slightly during the initial stages of shearing, and remains relatively constant thereafter. The degree of anisotropy also increases with sample density. This can broadly be related to the $q-\varepsilon_{a}$ response illustrated in Figure 2(a). In all cases the increase in $\phi_{d e v}$ reflects a reorientation of the contacts so that there are proportionally more contacts orientated in the direction of the major principal stress. Figure 7(b) also considers the variation of $\phi_{d e v}$ with $\varepsilon_{a}$, but in this case $\phi_{d e v}$ is determined by considering only the coarse-coarse contacts.

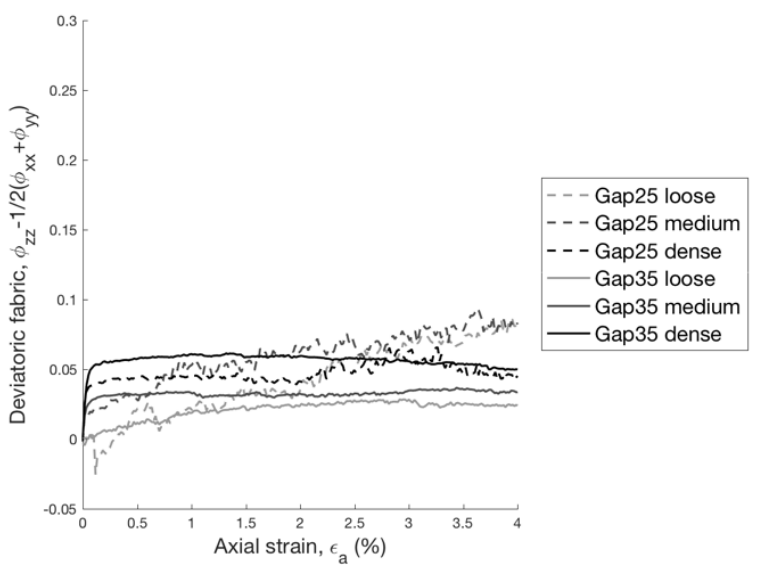

(a)

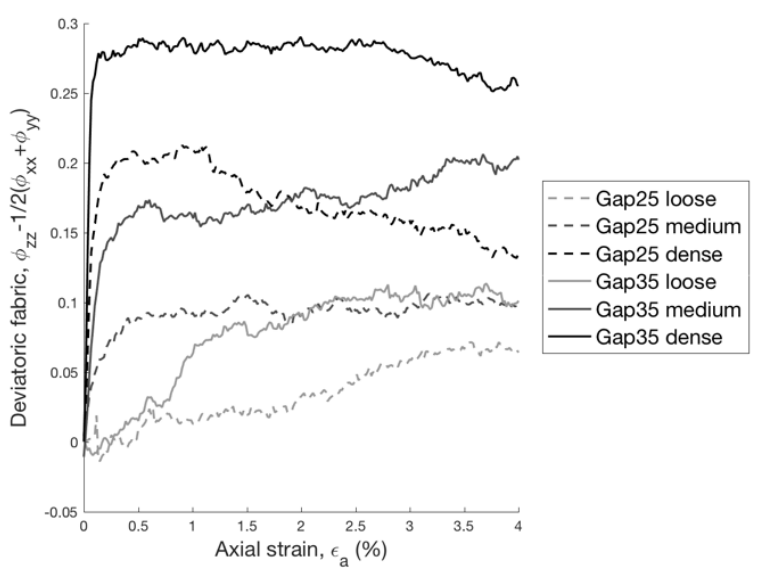

(b)

Fig. 7. Evolution of sample anisotropy with shearing (a) $\phi_{d e v}$ versus axial strain considering all contacts in sample (b) $\phi_{d e v}$ versus axial strain considering only coarse-coarse contacts 
Gap25 loose at $\epsilon_{\mathrm{a}}=4 \%$ with $\phi_{\mathrm{zz}}=0.387$

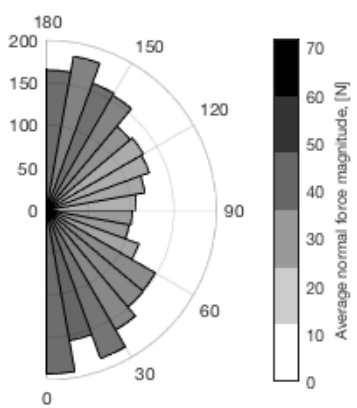

Gap35 loose at $\epsilon_{\mathrm{a}}=4 \%$ with $\phi_{\mathrm{zz}}=0.349$

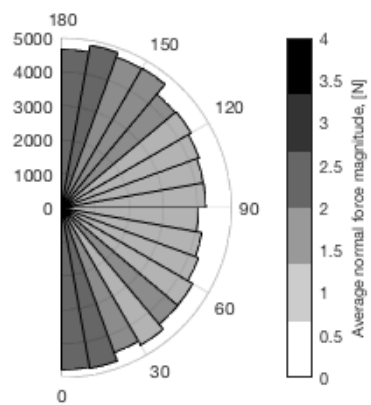

Gap25 medium at $\epsilon_{\mathrm{a}}=4 \%$ with $\phi_{\mathrm{zz}}=0.386$

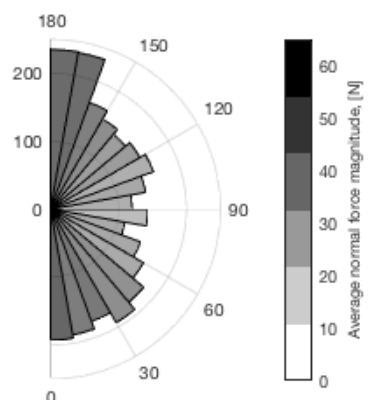

Gap35 medium at $\epsilon_{\mathrm{a}}=4 \%$ with $\phi_{\mathrm{zz}}=0.355$

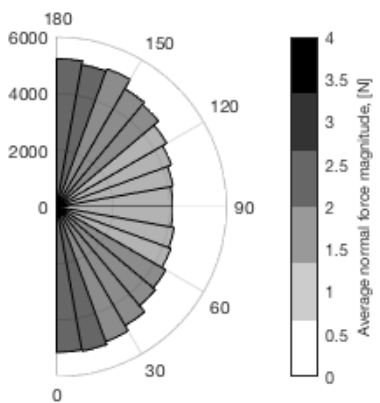

Gap25 dense at $\epsilon_{\mathrm{a}}=4 \%$ with $\phi_{\mathrm{zz}}=0.377$

180

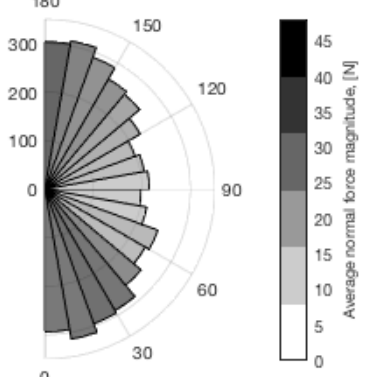

Gap35 dense at $\epsilon_{\mathrm{a}}=4 \%$ with $\phi_{\mathrm{zz}}=0.366$

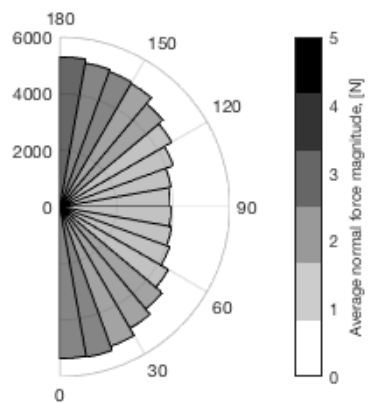

Fig. 8. Rose diagrams considering all contacts at an axial strain $\left(\varepsilon_{a}\right)$ of $4 \%$

Gap25 loose at $\epsilon_{\mathrm{a}}=4 \%$ with $\phi_{\mathrm{zz}}=0.38$

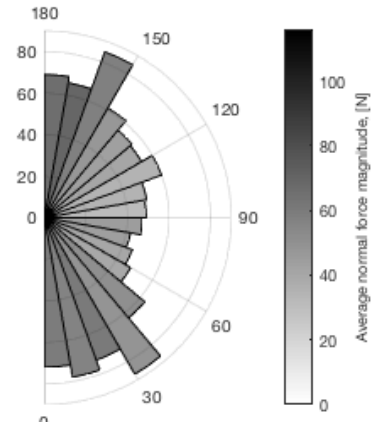

Gap35 loose at $\epsilon_{\mathrm{a}}=4 \%$ with $\phi_{\mathrm{zz}}=0.403$

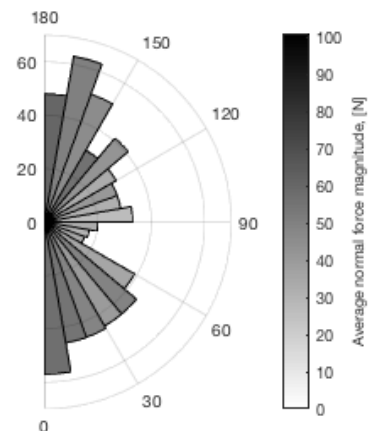

Gap25 medium at $\epsilon_{\mathrm{a}}=4 \%$ with $\phi_{\mathrm{zz}}=0.396$

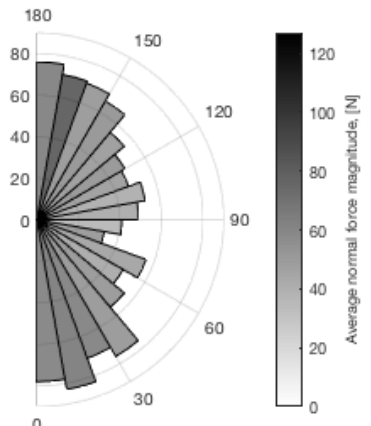

Gap35 medium at $\epsilon_{\mathrm{a}}=4 \%$ with $\phi_{\mathrm{zz}}=0.466$

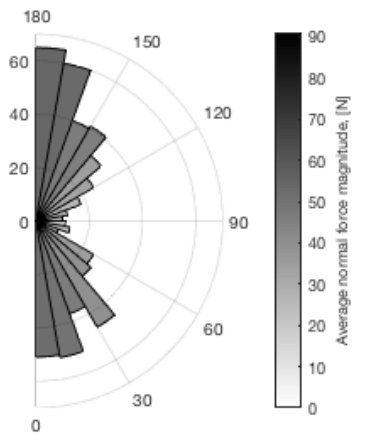

Gap25 dense at $\epsilon_{\mathrm{a}}=4 \%$ with $\phi_{\mathrm{zz}}=0.434$

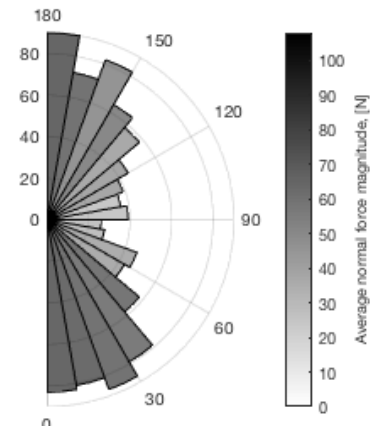

Gap35 dense at $\epsilon_{\mathrm{a}}=4 \%$ with $\phi_{\mathrm{zz}}=0.501$

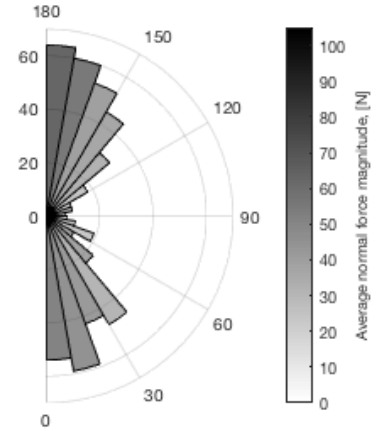

Fig. 9. Coarse-coarse contact rose diagrams at an axial strain $\left(\varepsilon_{a}\right)$ of $4 \%$

There is a measurable difference in the data presented in Figure 7(a) and Figure 7(b); for all of the Gap35 samples as well as for the Gap25 medium and dense samples the $\phi_{d e v}$ values calculated for the coarse-coarse contacts exceed the $\phi_{d e v}$ values calculated considering all of the contacts. This indicates that for these samples the imposition of shear deformation has a greater than average influence on the coarse-coarse contacts even in the case where $\alpha$ is high (as in sample Gap35 dense).

Further details are provided in the rose diagrams presented in Figures 8 and 9. The rose diagrams consider a projection of the contact force vectors on to the $x-z$ 
plane. The angular increment (bin size) is $10^{\circ}$ and each bin is shaded according to the average normal forces for contacts orientated within that interval. In all cases the diagrams consider the contact force network at the end of the simulation ( $\varepsilon_{a} \approx 4 \%$ ). Figure 8 considers all contacts in the system and Figure 9 considers only the coarse-coarse contacts. The smoother shape of the distributions observed for the Gap35 samples in comparison with the Gap25 samples reflects the differences in the numbers of active contacts discussed above.

It is clear from the diagrams presented in Figure 8 that there is an anisotropy in the force magnitudes as well as their orientations; the vertically orientated contacts exhibit larger forces, in agreement with the stress anisotropy. Contrasting Figures 8 and 9, a higher degree of preferential orientation of the coarse-coarse contacts in the vertical direction is clearly observed in comparison with the complete force network.

\section{Conclusions}

This study utilises DEM simulations to better understand the load:deformation response and the susceptibility to seepage-induced instability in gap-graded mixtures of sand/ gravel with non-plastic fines (silt/finer sand). The key observations can be summarised as follows:

1. The concept of a $F C_{t}$ that delineates sand and fines dominated behaviour has been considered in the literature. As discussed in Zuo and Baudet [3]different $F C_{t}$ values can be found using different rational experimental techniques. Developing upon the ideas of Skempton and Brogan [1], Shire et al. [2] proposed that rather than a single $F C_{t}$ value there is a range of $F C$ values, $F C^{*}<F C<F C^{+}$, for which the extent to which the fines participate in the mechanical behaviour is density-dependant. This contribution provides further evidence of the complexity associated with delineating fines-dominated and sand-dominated behaviour; a dilatant finesdominated material with $\alpha \geq 1$ can evolve to become a sand-dominated material with $\alpha<1$ due to shearing induced-dilation.

2. The significant decrease in $\alpha$ observed for the dense samples considered here indicates that in-situ the susceptibility to seepage induced internal instability may be increased due to deformation of embankments or their foundations.

3. The relationship between $\alpha$ and e is non-trivial, while $\alpha$ clearly reduces as $e$ increases, for a given grading the same $\alpha$ values can be associated with different $e$ values, depending on the initial void ratio. There appears to be a unique, but non-linear relationship, between $e_{s}$, the non-rattler void ratio, and $\alpha$.

4. For most of the samples considered in this study, the shear deformation produced a stress carrying structure dominated by coarse-coarse contacts aligning in the axial direction; while other contacts have a more isotropic distribution and can be considered to act as bracing supporting the dominant coarse-coarse contact network.
5. The sample sizes considered here (36,950 and 50,357 particles) are reasonably large when compared with other DEM studies published in the geomechanics literature. The fluctuations in the load deformation response are indicative of small sample sizes. These observations indicate that for gap-graded materials very large samples are needed to enable representative element volumes to be achieved with confidence.

This research was supported by EPSRC grant EP/P010393/1. Ms. Marion Artigaut's time on this research was supported by the British Dam Society.

\section{References}

1. A.W. Skempton, J.M. Brogan, Experiments on piping in sandy gravels. Géotechnique 44:565-567, (1994).

2. T. Shire, C.O' Sullivan, K.J. Hanley, R.J. Fannin, Fabric and Effective Stress Distribution in Internally Unstable Soils. J Geotech Geoenvironmental Eng 140:1-11, (2014).

3. L. Zuo, B.A. Baudet, Determination of the transitional fines content of sand-non plastic fines mixtures. Soils Found 55:213-219, (2015).

4. K. Kawano, T. Shire, C. O’Sullivan, Coupled particle-fluid simulations of the initiation of suffusion. Soils Found 58:972-985, (2018).

5. C. Thornton, Numerical simulations of deviatoric shear deformation of granular media. Géotechnique 50:43-53, (2000).

6. S. Plimpton, Fast Parallel Algorithms for ShortRange Molecular Dynamics. J Comput Phys 117:119. doi: 10.1006/jcph.1995.1039, (1995)

7. X. Huang, C. O’Sullivan, K.J. Hanley, F.C.Y. Kwok, Discrete-element method analysis of the state parameter. Géotechnique 64:954-965, (2014).

8. D. Barreto Gonzalez, Numerical and expeirmental investigation into the behaviour of granular materials under generalised stress states. University of London, (2009)

9. D.O. Potyondy, Simulating stress corrosion with a bonded-particle model for rock. Int J Rock Mech Min Sci 44:677-691, (2007).

10. S. Thevanayagam, T. Shenthan, S. Mohan, J. Liang, Undrained Fragility of Clean Sands, Silty Sands, and Sandy Silts. J Geotech Geoenvironmental Eng 128:849-859, (2002). 